\title{
Analysis of High-Risk Factors Associated with the Progression of Subaneurysmal Aorta to Abdominal Aortic Aneurysm in Rural Area in China
}

\author{
Wenjun Zhao' \\ Gang Wang ${ }^{2}$ \\ Ping $\mathrm{Xu}^{3}$ \\ Tingting $\mathrm{Wu}^{3}$ \\ Binjuan $\mathrm{Chen}^{3}$ \\ Haijun Ren ${ }^{2}$ \\ Xingjie $\mathrm{Li}^{1}$
}

'Department of Ultrasound, Imaging, Healthy Management Centre, The Second Hospital of Lanzhou University, Lanzhou City, 730000, Gansu Province, People's Republic of China; ${ }^{2}$ Department of Neurosurgeon, Neurology, Neurologist, The Second Hospital of Lanzhou University, Lanzhou City, 730000, Gansu Province, People's Republic of China; ${ }^{3}$ Department of Ultrasound, Imaging, Ultrasound Centre, The Second Hospital of Lanzhou University, Lanzhou City, 730000, Gansu Province, People's Republic of China
Correspondence: Xingiie Li Department of Ultrasound, Imaging Healthy Management Centre, The Second Hospital of Lanzhou University, Landa 2 Yard, No. 82, Cuiying Gate, Chengguan District, Lanzhou City, 730000, Gansu Province, People's Republic of China Tel +86-93 I8942555; +86-13609337887 Email LiXingjie723723@I63.com
Objective: To determine the risk factors associated with the progress of subaneurysmal aorta (SAA) to abdominal aortic aneurysm (AAA) and provide a reference for the prevention of AAA in rural areas.

Methods: A total of 747 SAA patients screened by the Health Management Center of the Second Hospital of Lanzhou University from January 2015 to January 2016 were recruited. The ratio of SAA progressing to AAA was observed through 5 years of follow-up. Logistic stepwise regression analysis was performed to analyze the high-risk factors. The relevant clinical prediction model score table (Nom) was made and the C-index and calibration chart were used to verify the prediction ability of the model.

Results: Of the 747 patients diagnosed with SAA, 260 developed to AAA, with an incidence of $34.8 \%$. Univariate analysis showed that age (62-65 years old), abdominal aorta diameter greater than $2.7 \mathrm{~cm}$, smoking after 30 years old, moderate to severe hypertension, and blood pressure variability were the important high-risk factors of SAA progressing to AAA. Logistic regression analysis showed that these factors were statistically significant. The nomogram of clinical prediction model score showed that when $50-60 \%$ of SAA developed to AAA, the score was $189-201$ and the C-index was 0.883 , verifying the moderate predictive ability of this model.

Conclusion: Age, smoking habit, degree of hypertension, and control situation were highrisk factors associated with the progression of SAA to AAA. The control of the above highrisk factors was imperative for the prevention of AAA in rural areas without sufficient medical resources.

Keywords: abdominal aortic aneurysm, high-risk factors, abdominal aorta dilation, rural areas

\section{Introduction}

Abdominal aortic aneurysm (AAA) is commonly defined as localized aortic aneurysmal or sac-like dilatation. AAA can be diagnosed when the diameter exceeds $3 \mathrm{~cm}$ or the vessel diameter in the lesion segment exceeds $50 \%$ of the normal diameter $(2 \mathrm{~cm})$ or is more than 1.5 times the normal vessel diameter adjacent to the distal end. ${ }^{1}$ If early diagnosis and treatment are not available, about $30 \%$ of patients will have AAA rupture. Once rupture occurs, only $21 \%$ to $33 \%$ of patients can survive., Subaneurysmal aortic dilatation (subaneurysmal aorta, SAA) refers to the diameter 
of the abdominal aorta between 2.6 and $2.9 \mathrm{~cm}$, that is, the diameter of the abdominal aorta is significantly larger than that of the normal, but has not yet reached the diagnostic criteria of AAA. ${ }^{4}$ The previous study has shown that $67 \%$ of SAA progresses to AAA during the 5-year follow-up. ${ }^{5}$ Hence, exploring and controlling the high-risk factors associated with the progress of SAA to AAA are of great significance for the prevention of AAA.

SAA is currently considered as the prototype of AAA, therefore, most studies on SAA worldwide are included in the screening of AAA, and the population selection is synchronized with AAA, the overall population aged 6575 years old. ${ }^{6,7}$ However, the incidence of SAA varies in different age groups. The incidence of AAA among smoking men aged $65-74$ years is $8.2 \%$, and that of smoking men aged 65 years is $2.1 \%{ }^{8}$ Importantly, there still lacks studies on the risk factors of SAA progressing to AAA in rural areas. Therefore, this study investigated the incidence of SAA among 60-65-year-old smoking men in 3 rural areas around Lanzhou city. After about 5 years of followup, their outcomes and related high-risk factors were explored. To explore the significance of SAA screening in rural areas of northwest China and to highlight the control of these high-risk factors are crucial for the prevention of abdominal aortic aneurysm in rural areas with insufficient medical resources.

\section{Materials and Methods General Information}

Totally 836 patients with subaneurysmal aortic dilatation screened by the Health Management Center of the Second Hospital of Lanzhou University from January 2015 to January 2016 were included after the 5-year follow-up in 2019. Inclusion criteria were as follows: (1) smoking male; (2) 60-65 years old; (3) living in three rural areas around Lanzhou city; (4) abdominal aorta confirmed as SAA by ultrasound; (5) all signed informed consent and voluntarily participated in this study. The patients with special infectious diseases, connective tissue diseases, rheumatic immune diseases, severe consciousness disorder, and dysfunction of important organs such as heart, liver, and kidney were excluded. 765 SAA patients participated rescreening and 747 valid measurements were adopted.

\section{Methods}

The data of the population undergoing physical examination at the Health Management Center of the Second
Hospital of Lanzhou University from January 2015 to January 2016 were retrieved. The first ultrasound examination results of abdominal aorta in 60-65 years old male smokers living in three rural areas around Lanzhou city were collected. The physical examinees who were diagnosed as SAA were screened out. SAA was defined as the diameter of the abdominal aorta between 2.6 and $2.9 \mathrm{~cm}$, which is significantly larger than normal but smaller than AAA. Here, the diameter refers to the width from the anterior wall of abdominal aorta renal artery branch to the inner edge of the posterior wall. Five years later, in 2019, the above physical examiners were contacted by telephone to come to our center for the second reexamination. Two professional sonographers used the IU 22 ultrasound instrument to complete the examination order to reduce the division error. The subjects who finally completed the reexamination were investigated through a questionnaire survey under the guidance of the staff of the center, including personal basic information, smoking habits, blood pressure, understanding of AAA-related information, whether to choose intervention treatment and selection reasons. The overall data were statistically analyzed to make the relevant clinical prediction model score table (Nom), and the C-index and Calibration chart were used to verify the prediction ability of the model. The study flow chart is shown in Figure 1.

\section{Data Entry}

A retrospective study was used. Two resident physicians who did not participate in the data analysis respectively checked the patients' medical records at the time of data entry and recorded the clinical data of patients including gender, age, SAA diameter, hypertension status (mild: systolic pressure (SP) $140-159 \mathrm{mmHg}$ or diastolic pressure (DP) $90-99 \mathrm{mmHg}$; moderate: SP $160-179 \mathrm{mmHg}$ or DP 100-109mmHg; severe: SP $\geq 180 \mathrm{mmHg}$ or DP $\geq 110 \mathrm{mmHg}$ ), blood pressure variability (excess the range of SP $90-139 \mathrm{mmHg}$ and DP $60-89 \mathrm{mmHg}$ ), smoking status, and any other clinical diseases or not. The data were entered uniformly after verification. If there was any doubt, it shall be discussed by the chief physician of the third party to reach an agreement.

\section{Statistical Methods}

Statistical evaluation was performed using R Studio software. All data were described as mean \pm standard deviation (SD) if applicable. Logistic stepwise regression analysis was performed to analyze the associations 


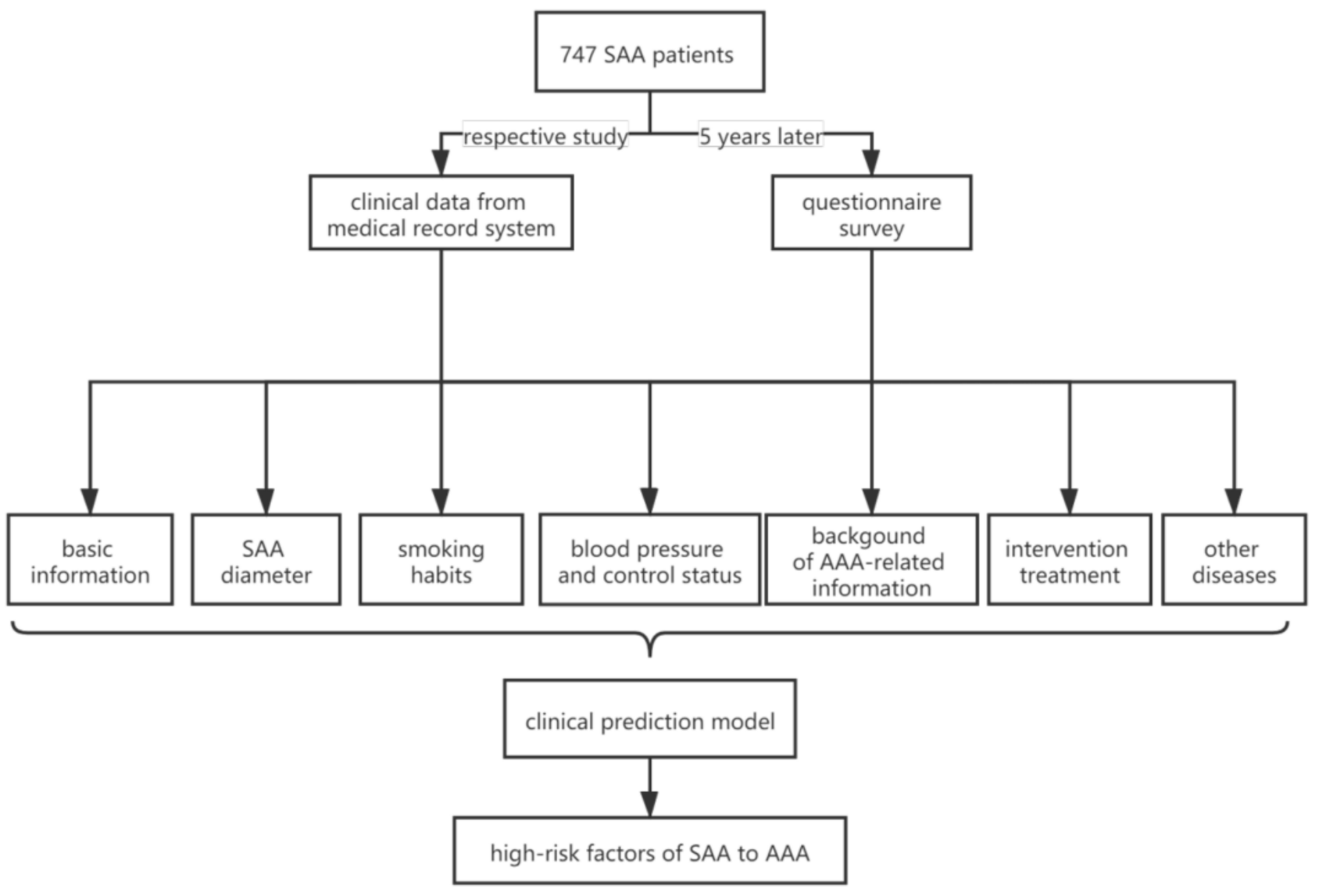

Figure I Study flow chart.

among different parameters. A value of $\mathrm{P}<0.05$ was considered statistically significant and $95 \%$ confidence interval (CI).

\section{Results}

\section{The Rate of SAA Patients Progressing to}

\section{AAA Was 34.8\%}

We collected the first ultrasound examination results of abdominal aorta of 22,841 smoking men aged 60-65 years old in the surrounding counties and towns of Lanzhou city. Basic demographics of patients are shown in Table 1. Among them, 836 were diagnosed as SAA (3.66\%) and 765 SAA patients participated in the reexamination, with a participation rate of $91.5 \%$. Finally, 747 valid measurements were adopted (97.6\%). After a 5-year follow-up, 71 SAA patients were withdrawn $(8.5 \%)$, of whom 48 were lost to contact, 12 were died of disease (5 from cerebral hemorrhage, 1 from pancreatic cancer, 1 from lung cancer, 1 from prostate cancer, 1 from respiratory failure, 1 from gastric cancer, 1 from colon cancer, and 1 from accidental death), 11 patients dropped out of screening. Among them, 260 SAA were diagnosed as AAA in 2019. Overall, the rate of SAA patients progressing to AAA was $34.8 \%$.

\section{Univariate Analysis Showed That Smoking, Moderate to Severe Hypertension, and Blood Pressure Variability Were the High-Risk Factors of SAA Progressing to AAA}

Univariate analysis showed that age (62-65 years old), abdominal aorta diameter greater than $2.7 \mathrm{~cm}$, smoking after 30 years old, moderate to severe hypertension, and blood pressure variability were important high-risk factors of SAA progressing to AAA (Table 2).

\section{Multivariate Analysis of the High-Risk Factors of SAA Progressing to AAA}

Logistic regression model analysis showed that $62-65$ years old $(\mathrm{OR}=2.965)$, primary abdominal aorta diameter 
Table I Basic Demographics of Patients

\begin{tabular}{|c|c|c|c|c|c|c|c|}
\hline Age & 60 & 61 & 62 & 63 & 64 & 65 & Total \\
\hline \multicolumn{8}{|l|}{ Screening } \\
\hline $2.6 \mathrm{~cm}$ & 36 & 39 & 32 & 36 & 34 & 29 & 206 \\
\hline $2.7 \mathrm{~cm}$ & 14 & 32 & 36 & 42 & 35 & 31 & 190 \\
\hline $2.8 \mathrm{~cm}$ & 18 & 30 & 35 & 47 & 42 & 44 & 216 \\
\hline $2.9 \mathrm{~cm}$ & 16 & 24 & 33 & 42 & 51 & 58 & 224 \\
\hline Total & 84 & 125 & 136 & 167 & 162 & 162 & 836 \\
\hline \multicolumn{8}{|l|}{ Rescreening } \\
\hline SAA & 78 & 119 & 121 & 149 & 156 & 142 & 765 \\
\hline AAA & 21 & 17 & 27 & 53 & 43 & 99 & 260 \\
\hline \multicolumn{8}{|c|}{ Smoke pack/day } \\
\hline$<$ Ipack & 9 & 4 & 8 & 6 & 2 & 13 & 42 \\
\hline I-2packs & 55 & 36 & 46 & 53 & 41 & 82 & 313 \\
\hline 2-3packs & 56 & 17 & 49 & 51 & 51 & 83 & 307 \\
\hline$>3$ packs & 14 & 8 & 10 & 20 & 9 & 24 & 85 \\
\hline \multicolumn{8}{|l|}{ Start smoking } \\
\hline$<20$ age & 20 & 4 & 26 & 31 & 21 & 21 & 123 \\
\hline 20-25age & 60 & 13 & 56 & 48 & 24 & 86 & 287 \\
\hline 26-30age & 44 & 30 & 26 & 45 & 50 & 75 & 270 \\
\hline$>30$ age & 10 & 18 & 5 & 7 & 8 & 19 & 67 \\
\hline \multicolumn{8}{|l|}{$\mathbf{H}$} \\
\hline Norm & 50 & 20 & 23 & 20 & 22 & 38 & 173 \\
\hline Light & 47 & 25 & 27 & 48 & 22 & 65 & 234 \\
\hline Medium & 26 & 10 & 46 & 47 & 20 & 60 & 209 \\
\hline Heavy & 9 & 6 & 10 & 12 & 25 & 29 & 91 \\
\hline Fluctuate & 2 & 4 & 7 & 3 & 14 & 10 & 40 \\
\hline \multicolumn{8}{|c|}{ AAA knowledge } \\
\hline Yes & 2 & 2 & 3 & I & 2 & 1 & II \\
\hline No & 132 & 63 & 110 & 129 & 101 & 201 & 736 \\
\hline \multicolumn{8}{|c|}{ Intervene treatment } \\
\hline Accept & 38 & 40 & 94 & 93 & 92 & 178 & 535 \\
\hline Refuse & 66 & 25 & 39 & 27 & 11 & 44 & 212 \\
\hline \multicolumn{8}{|c|}{ Refuse treatment reason } \\
\hline Expensive price & 110 & 60 & 103 & 100 & 90 & 190 & 653 \\
\hline Other & 24 & 5 & 10 & 30 & 13 & 12 & 94 \\
\hline
\end{tabular}

greater than $2.7 \mathrm{~cm}(\mathrm{OR}=2.828)$, smoking after 30 years old $(\mathrm{OR}=2.783)$, moderate hypertension $(\mathrm{OR}=2.63)$, severe hypertension $(\mathrm{OR}=2.917)$, and blood pressure variability $(\mathrm{OR}=3.164)$ had significant statistical significance (Figure 2).
Table 2 The Correlation Between Continuous Variables and the Progression of SAA to AAA

\begin{tabular}{|c|c|c|c|}
\hline item & $\begin{array}{l}\text { Standard } \\
\text { Deviation }\end{array}$ & $\begin{array}{l}\text { Standard } \\
\text { Error }\end{array}$ & $p$ \\
\hline \multicolumn{4}{|l|}{ Age } \\
\hline 61 & 0.66158 & 0.59795 & 0.268548 \\
\hline 62 & 2.03359 & 0.49235 & $3.62 \mathrm{e}-05^{* *}$ \\
\hline 63 & 2.96665 & 0.47064 & $2.91 \mathrm{e}-10 * *$ \\
\hline 64 & 2.19817 & 0.48314 & $5.37 e-06 * *$ \\
\hline 65 & 2.4582 & 0.44337 & $2.95 \mathrm{e}-08 * *$ \\
\hline \multicolumn{4}{|c|}{ Abdominal aorta diameter } \\
\hline $2.7 \mathrm{~cm}$ & -2.09268 & $0.6334 I$ & $0.000954^{* *}$ \\
\hline $2.8 \mathrm{~cm}$ & -0.78781 & 0.575 & 0.170652 \\
\hline $2.9 \mathrm{~cm}$ & 0.38843 & 0.60257 & 0.519175 \\
\hline \multicolumn{4}{|l|}{ Smoking } \\
\hline I-2pack & -0.13204 & 0.50269 & 0.792815 \\
\hline 2-3pack & 0.03557 & 0.50663 & 0.94402 \\
\hline >3pack & 0.46208 & 0.54015 & 0.392291 \\
\hline \multicolumn{4}{|c|}{ Age of smoking initiation } \\
\hline $20-25$ & -0.61222 & 0.29853 & $0.040290 *$ \\
\hline $26-30$ & 0.54992 & 0.29647 & 0.063614 \\
\hline$>30$ & 2.87224 & $0.5173 \mid$ & $2.82 \mathrm{e}-08 * *$ \\
\hline \multicolumn{4}{|c|}{ Degree of hypertension } \\
\hline Mild & 0.55412 & 0.31496 & 0.07852 \\
\hline Moderate & 2.25794 & 0.36163 & $4.27 \mathrm{e}-10 * *$ \\
\hline Severe & 2.91646 & 0.41738 & $2.80 \mathrm{e}-12 * *$ \\
\hline $\begin{array}{l}\text { Blood pressure } \\
\text { fluctuations }\end{array}$ & 1.88942 & 0.54409 & $0.000515 * *$ \\
\hline Knowing & -0.32664 & 0.25788 & 0.205282 \\
\hline Intervention & 0.24132 & 0.28624 & 0.399183 \\
\hline Economic factors & 0.32682 & 0.22157 & 0.140202 \\
\hline
\end{tabular}

Note: $* P<0.05$ is considered to be statistically significant, $* * P<0.01$ is considered to be obvious statistically significant, "e" means exponent.

\section{The Prediction Score of the Clinical Prediction Model}

The meaningful risk factors were screened out through the $\lambda$ coefficient, and the relevant clinical prediction model score table (Nom) was made. The C-index and Calibration chart were used to verify the predictive ability of this model (Figure 3, Table 3). The predictive equation was shown as follows: when se $<-0.067 / 2,11<-0.883+1.96 * \mathrm{se}$, $0.883+1.96 * \mathrm{se}$; when $\mathrm{se}<-0.067 / 2, \quad 1 \mathrm{l}<-0.883-1.96^{*} \mathrm{se}$, $0.883-1.96 * \mathrm{se}, 95 \%$ CI: $0.81734-0.94866$. 
A

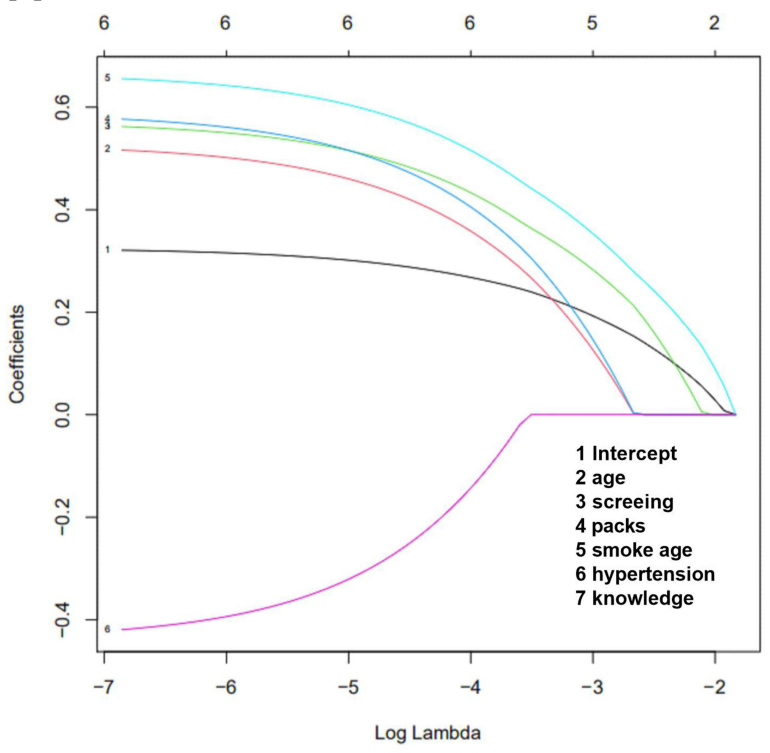

B

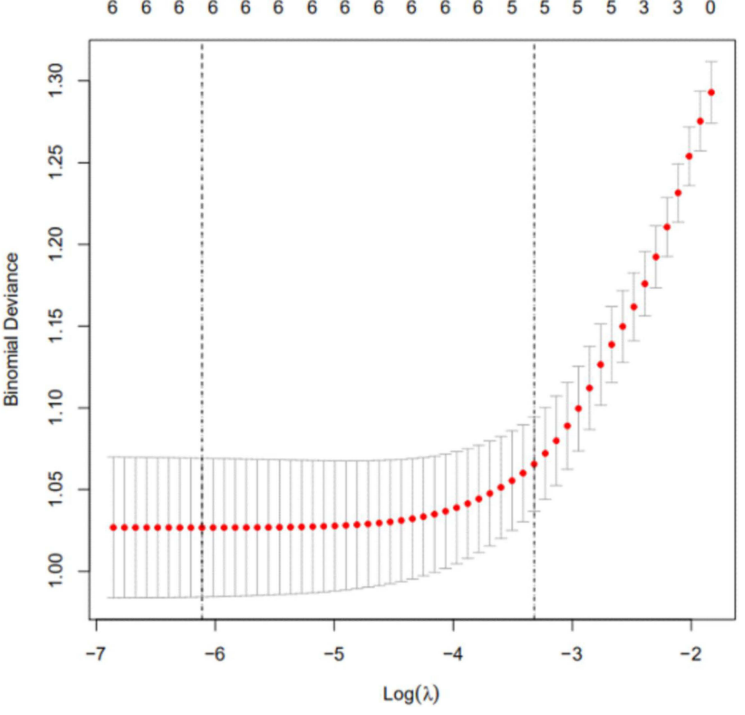

Figure 2 The correlation between variables and the progress of SAA to AAA. Multivariate analysis of the high-risk factors of SAA progressing to AAA. (A) Verification diagram of SAA-related risk factors; (B) screening diagram of SAA-related risk factors.

Points

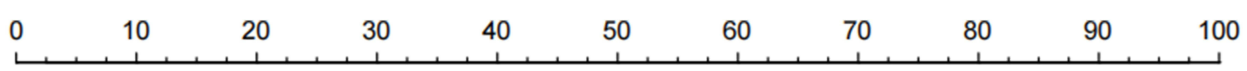

age

\begin{tabular}{|c|c|c|c|}
\hline & 61 & & 84 \\
\hline \multirow[t]{2}{*}{60} & & 62 & 65 \\
\hline & $2.8 \mathrm{~cm}$ & & $2.9 \mathrm{~cm}$ \\
\hline $2.7 \mathrm{~cm}$ & & $2.6 \mathrm{~cm}$ & \\
\hline 2-3pack & >3pack & & \\
\hline
\end{tabular}

smoke_age

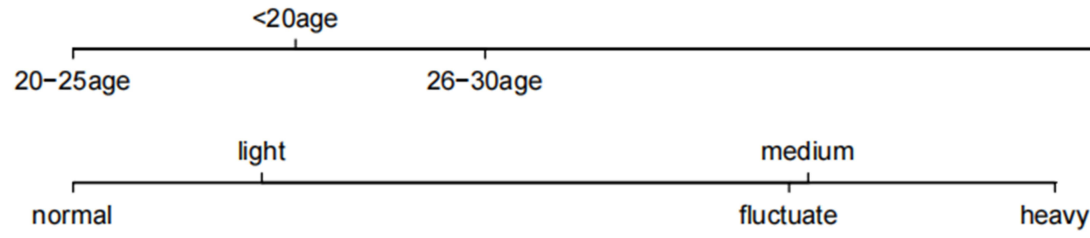

knowledge

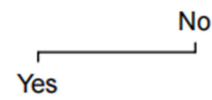

Total Points

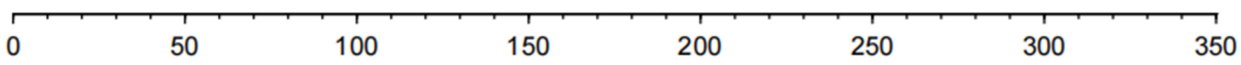

Risk of AAA

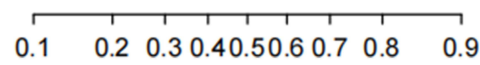

Figure 3 Clinical prediction model prediction score. The C-index was performed to verify the predictive ability. 
Table 3 The Probability of Developing AAA with the Total Score of Risk Factors

\begin{tabular}{|l|c|}
\hline $\begin{array}{l}\text { Total Risk Factor } \\
\text { Score }\end{array}$ & Probability of Progressing to AAA (\%) \\
\hline 127 & 10 \\
150 & 20 \\
165 & 30 \\
178 & 40 \\
189 & 50 \\
201 & 60 \\
213 & 70 \\
228 & 80 \\
251 & 90 \\
\hline
\end{tabular}

The nomogram of the prediction score of the clinical prediction model showed that when $50-60 \%$ of SAA developed to AAA, the score was 189-201 and the C-index was 0.883 , which proved that this model had the moderate predictive ability. The Calibration chart also showed a good fit with the ideal state (Figure 4, Table 4).

\section{Discussion}

The European Society of Vascular Society (ESVS) in 2019 and the American Society of Vascular Surgery (ASVS) in 2018 recommended that the diameter changes of dilation in SAA patients should be monitored every 5-10 years, ${ }^{9,10}$ but the clear specifications on the guidelines for screening age range have not been made. The current studies mostly choose the SAA screening age in the range of 65-75 years

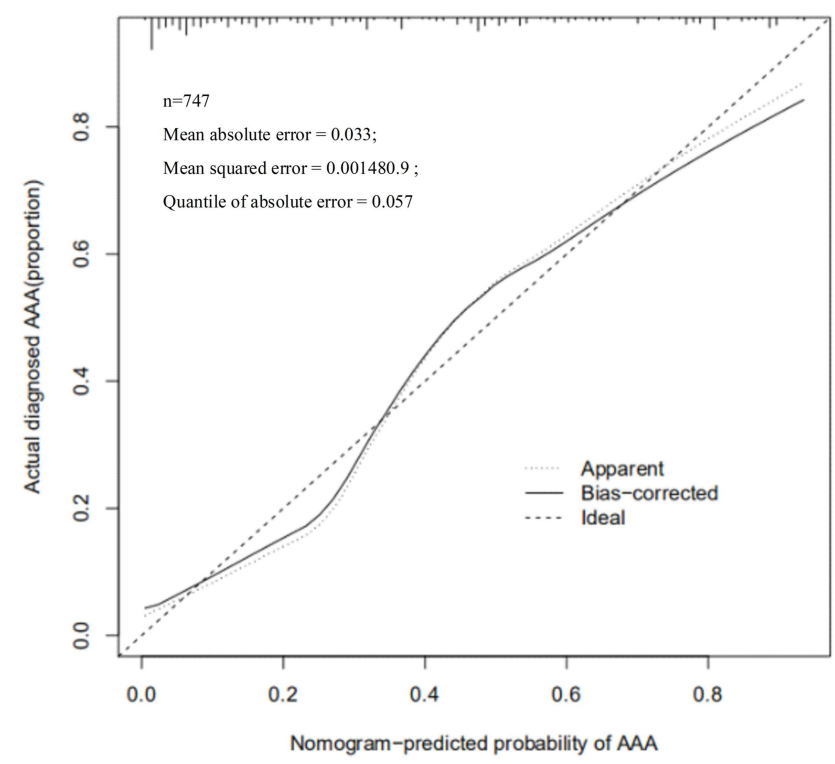

Figure 4 Verification of the predictive ability of the model. The Nomogrampredicted probability of AAA were determined by Calibration chart.
Table 4 The Predictive Ability of the Verification Model

\begin{tabular}{|l|c|c|c|c|}
\hline Model & Likelyhood & $\begin{array}{c}\text { Discrimination } \\
\text { Ratio Test }\end{array}$ & $\begin{array}{c}\text { Rank } \\
\text { Discrim. } \\
\text { Indexes }\end{array}$ & Indexes \\
\hline Obs & 747 & LR chi2 329.88 & R2 0.492 & c 0.883 \\
\hline 0 & 487 & d.f. 21 & g 2.388 & $\begin{array}{c}\text { Dxy } \\
0.744\end{array}$ \\
\hline I & & $\begin{array}{c}\text { Pr(>chi2) } \\
<0.000 \text { I }\end{array}$ & gr 10.887 & $\begin{array}{c}\text { Gamma } \\
0.744\end{array}$ \\
\hline
\end{tabular}

old, ${ }^{11,12}$ which is the same as the recommended age range for AAA screening. However, since SAA is an important stage before the formation of AAA, this study advanced SAA screening age range.

Our study found that the incidence rate of SAA in smoking men aged 60-65 years old in three rural areas around Lanzhou city was $3.66 \%$, which is lower than that of the same age group reported in foreign literature. ${ }^{13,14}$ The reason may be that the incidence of AAA in Asia is lower than that in European and American area and AAA develops from SAA. Hence, the incidence of SAA in Asia is also lower than that in European and American areas. In addition, it is reported that $52-67 \%$ of 65 -year-old SAA patients develop to AAA after 5 years. In this study, $40 \%$ of 63-year-old SAA progressed to AAA after 5 years and the progress rate reached $65 \%$ in 65 -year-old SAA patients, which is consistent with the foreign studies. It can be seen that although the incidence of SAA in smoking males aged 65 years in this study is lower than that in the European and American areas, the rate of developing AAA after 5 years is basically the same as that of foreign countries, which may be closely related to the effects of high-risk factors.

Through the $\lambda$ model, we find that there were other meaningful risk factors besides age, and smoking was the first high-risk factor. Studies have shown that 65-year-old SAA patients have a $60 \%$ chance of progressing to AAA if they smoke. ${ }^{15,16}$ Therefore, smoking as the most important independent risk factor exerts great effects on the occurrence and development of SAA. Our results showed that more than $50 \%$ of SAA patients smoked more than 2-3 packets, and the more packets smoked per day and the later they start smoking ( $>30$ years old), the more statistically significant smoking as a risk factor of SAA. SAA patients can benefit from smoking cessation. ${ }^{17,18}$ There are a large number of smokers in rural areas of northwest 
China and the smoking habit has lasted for a long time. ${ }^{19}$ Therefore, the supervision measures for smoking cessation should be implemented in rural areas to reduce the rate of SAA progressing to AAA. Moreover, studies at home and abroad have shown that hypertension is closely related to the progress of AAA. Moderately to severely elevated blood pressure, poor control, and large variability all contribute to the development of AAA. ${ }^{20,21}$ This study also found that hypertension was a high-risk factor of SAA progressing to AAA. Particularly, moderate to severe hypertension and blood pressure variability significantly promoted the progress of SAA to AAA, which is consistent with the results of foreign studies. For the SAA patients diagnosed at the age of $65,26.2-28 \%$ of patients developed to AAA with a diameter $\geq 5.5 \mathrm{~cm}$ and surgical indications after 10-15 years. Unfortunately, the questionnaire results demonstrated that SAA patients basically did not have the understanding of AAA-related information. Even after being taught about the risk of AAA, these patients still preferred to choose conservative treatment rather than surgical intervention. The main reason for such a choice was the cost of treatment. Therefore, the demand for routine AAA screening in rural areas in the northwest is greater than the need for surgery and treatment. The residents in rural areas with economic underdevelopment are more benefited from screening.

Nomogram can integrate multiple clinical parameters and achieve individual prediction, which is a calculation chart that can replace complex mathematical formulas. It can present the results of regression analysis in an intuitive graphical form and has been widely used in the prediction of high-risk factors of many diseases, which is of great significance for the individual and accurate prognosis prediction. ${ }^{22}$ In this study, based on the above risk factors, we made a nomogram prediction model for SAA progressing to AAA. The relatively high scores among the single risk factors were 63 years old (85 points), SAA diameter of $2.9 \mathrm{~cm}$ (70 points), daily smoking over 3 packets (18 points), start smoking 30 years old (100 points), moderate to severe hypertension (62-82 points), and lack of AAA-related information (10 points). If $50-60 \%$ of SAA developed to AAA, the score was 189-201. It was suggested that as long as the score exceeded 60 points in a single risk factor, the progress probability of SAA meeting 3-4 risk factors to AAA would exceed 50\%. Especially smoking and hypertension accounted for a large score, reflecting the importance of controlling these two factors. The predictive ability of the model was further verified through $\mathrm{C}$-index. Generally, $0.7-$
0.9 is considered as a medium predictive ability. The C-index of this model was 0.883 , which proved that this model had a superior predictive ability. Calibration chart shows the predictive ability of the model graphically. Although some parts were in the ideal state, the overall fit of the curve was better. Notably, we were the first to use $\mathrm{R}$ Studio statistical software to screen the risk factors of SAA progressing to AAA, and further selected the statistically significant risk factors to draw the nomogram and calculate the score of each risk factor. This statistical method was first introduced to the analysis of high-risk factors of SAA progressing to AAA, which can avoid many subjective factors. There are some limitations in this study, other factors such as gender and diabetes may also have an effect on the progression of SAA to AAA, further studies are warranted to further elucidate the relationship involved.

\section{Conclusion}

We firstly combined $\mathrm{R}$ Studio with nomogram in the analysis of high-risk factors of SAA progressing to AAA. Our study elucidated that age, smoking habits, degree of hypertension, and control condition were the high-risk factors associated with the progression of SAA to AAA. The emphasis on the control of the abovementioned high-risk factors is of great significance for the prevention of AAA in rural areas lacking medical resources. SAA screening is the fundamental measure to reduce its development to AAA from the source, which has strong feasibility and superior clinical application value. However, we cannot copy the foreign screening models. At present, there are still no reports on SAA screening in China. Therefore, it is necessary to reformulate the screening process according to the actual needs in rural areas to achieve better results.

\section{Abbreviations}

SAA, subaneurysmal aorta; AAA, Abdominal aortic aneurysm; CI, confidence interval; ESVS, European Society of Vascular Society.

\section{Ethics Approval and Consent to Participate}

All the participants had signed informed consent. This study was designed in accordance with the Declaration of Helsinki and approved by the Ethics Committee of the Second Hospital of Lanzhou University. 


\section{Acknowledgment}

We would like to acknowledge the reviewers for their helpful comments on this paper. Wenjun Zhao and Gang Wang are co-first authors.

\section{Funding}

This research was supported by funds from the Ultrasound screening and grouping follow up of high-risk population of abdominal aortic aneurysm in Lanzhou (No: 18JR3RA324).

\section{Disclosure}

The authors declare that they have no conflicts of interest.

\section{References}

1. Golledge J. Abdominal aortic aneurysm: update on pathogenesis and medical treatments. Nat Rev Cardiol. 2019;16(4):225-242. doi:10.1038/s41569-018-0114-9

2. Memon AA, Zarrouk MZ, Ågren-witteschus S, Sundquist J, Gottsäter A, Sundquist K. Identification of novel diagnostic and prognostic biomarkers for abdominal aortic aneurysm. Eur J Prev Cardiol. 2020;27(2):132-142. doi:10.1177/2047487319873062

3. Brian Keisler CC. Abdominal aortic aneurysm. Am Fam Physician. 2015;91(8):538-543.

4. Stather PW, Dattani N, Bown MJ, Earnshaw JJ, Lees TA. International variations in AAA screening. Eur J Vasc Endovasc Surg. 2013;45 (3):231-234. doi:10.1016/j.ejvs.2012.12.013

5. Thorbjørnsen K, Svensjö S, Djavani Gidlund K, Gilgen N-P, Wanhainen A. Prevalence and natural history of and risk factors for subaneurysmal aorta among 65-year-old men. Ups J Med Sci. 2019;124(3):180-186. doi:10.1080/03009734.2019.1648611

6. Oliver-Williams C, Sweeting MJ, Turton G, et al. Gloucestershire and swindon abdominal aortic aneurysm screening programme. Lessons learned about prevalence and growth rates of abdominal aortic aneurysms from a 25 -year ultrasound population screening programme. $\mathrm{Br}$ J Surg. 2018;105(1):68-74. doi:10.1002/bjs.10715

7. Roddy SP. Abdominal aortic aneurysm screening. J Vasc Surg. 2017;65(5):1537. doi:10.1016/j.jvs.2017.02.021

8. Hamel C, Ghannad M, McInnes MD, et al. Potential benefits and harms of offering ultrasound surveillance to men aged 65 years and older with a subaneurysmal $(2.5-2.9 \mathrm{Cm})$ infrarenal aorta. J Vasc Surg. 2018;67(4):1298-1307. doi:10.1016/j.jvs.2017.11.074

9. Riambau V, Böckler D, Brunkwall J, et al. Editor's choice Management of Descending thoracic aorta diseases: clinical practice guidelines of the European Society for Vascular Surgery (ESVS). Eur J Vasc Endovasc Surg. 2017;53(1):4-52.
10. Earnshaw JJ, Lees T. Update on screening for abdominal aortic aneurysm. Eur J Vasc Endovasc Surg. 2017;54(1):1-2. doi:10.1016/ j.ejvs.2017.04.002

11. Zucker EJ, Prabhakar AM. Abdominal aortic aneurysm screening: concepts and controversies. Cardiovasc Diagn Ther. 2018;8(Suppl 1): S108-S117. doi:10.21037/cdt.2017.09.13

12. Bath MF, Sidloff D, Saratzis A, Bown MJ; UK Aneurysm Growth Study investigators. Impact of abdominal aortic aneurysm screening on quality of life. Br J Surg. 2018;105(3):203-208. doi:10.1002/ bjs. 10721

13. Ying AJ, Affan ET. Abdominal aortic aneurysm screening: a systematic review and meta-analysis of efficacy and cost. Ann Vasc Surg. 2019;54:298-303. doi:10.1016/j.avsg.2018.05.044

14. Wanhainen A, Verzini F, Van Herzeele I, et al. Editor's Choice European Society for Vascular Surgery (ESVS) 2019 clinical practice guidelines on the management of abdominal aorto-iliac artery aneurysms. Eur J Vasc Endovasc Surg. 2019;57(1):8-93.

15. Bohlin S, Fröjd C, Wanhainen A, Björck M. Change in smoking habits after having been screened for abdominal aortic aneurysm. Eur $J$ Vasc Endovasc Surg. 2014;48(2):138-143. doi:10.1016/j. ejvs.2014.04.010

16. Jahangir E, Lipworth L, Edwards TL, et al. Smoking, sex, risk factors and abdominal aortic aneurysms: a prospective study of 18782 persons aged above 65 years in the Southern Community Cohort Study. J Epidemiol Community Health. 2015;69(5):481-488. doi:10.1136/ jech-2014-204920

17. Saratzis A, Dattani N, Brown A, et al.; Vascular and Endovascular Research Network (VERN). Multi-centre study on cardiovascular risk management on patients undergoing AAA surveillance. Eur $J$ Vasc Endovasc Surg. 2017;54(1):116-122. doi:10.1016/j. ejvs.2017.04.009

18. Sakalihasan N, Michel JB, Katsargyris A, et al. Abdominal aortic aneurysms. Nat Rev Dis Primers. 2018;4(1):34.

19. Lei C, Wenxia L, Bing L. Investigation on the current status of smoking and drinking among the elderly in urban and rural areas in northwestern Hubei. Chin Gen Pract. 2013;11(5):764-765.

20. Elsa Kobeissi E, Pan M, Pan H, Aune D. Blood pressure, hypertension and the risk of abdominal aortic aneurysms: a Systematic Review and Meta-Analysis of Cohort Studies. Eur J Epidemiol. 2019;34(6):547-555. doi:10.1007/s10654-019-00510-9

21. Howard DPJ, Banerjee A, Fairhead JF, Handa A, Silver LE, Rothwell PM; Oxford Vascular Study. Age-specific incidence, Risk factors and outcome of acute abdominal aortic aneurysms in a defined population. Br J Surg. 2015;102(8):907-915. doi:10.1002/bjs.9838

22. Jelovsek JE, Chagin K, Brubaker L, et al.; Pelvic Floor Disorders Network. A model for predicting the risk of de novo stress urinary incontinence in women undergoing pelvic organ prolapse surgery. Obstet Gynecol. 2014;123(2 Pt 1):279-287. doi:10.1097/ AOG.0000000000000094
Clinical Interventions in Aging

\section{Publish your work in this journal}

Clinical Interventions in Aging is an international, peer-reviewed journal focusing on evidence-based reports on the value or lack thereof of treatments intended to prevent or delay the onset of maladaptive correlates of aging in human beings. This journal is indexed on PubMed Central, MedLine, CAS, Scopus and the Elsevier
Bibliographic databases. The manuscript management system is completely online and includes a very quick and fair peer-review system, which is all easy to use. Visit http://www.dovepress.com/ testimonials.php to read real quotes from published authors. 